\title{
The characteristics of host lipid body biogenesis during coral- dinoflagellate endosymbiosis
}

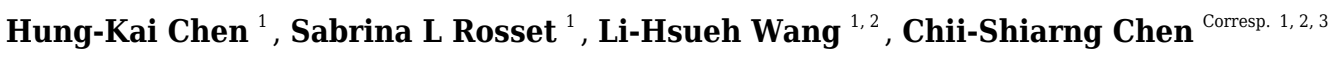 \\ 1 National Museum of Marine Biology and Aquarium, Pingtung, Taiwan \\ 2 Graduate Institute of Marine Biology, National Dong-Hwa University, Pingtung, Taiwan \\ 3 Department of Marine Biotechnology and Resources, National Sun Yat-sen University, Kaohsiung, Taiwan \\ Corresponding Author: Chii-Shiarng Chen \\ Email address: cchen@nmmba.gov.tw
}

Intracellular lipid body (LB) biogenesis depends on the symbiosis between coral hosts and their Symbiodinaceae. Therefore, understanding the mechanism(s) behind LB biosynthesis in corals can portentially elucide the drivers of cellular regulation during endosymbiosis. This study assessed LB formation in the gastrodermal tissue layer of the hermatypic coral Euphyllia glabrescens. Diel rhythmicity in LB size and distribution was observed; solar irradiation onset at sunrise initiated an increase in LB formation, which continued throughout the day and peaked after sunset at 18:00. The LBs migrated from the area near the mesoglea to the gastrodermal cell border near the coelenteron. Micro-LB biogenesis occurred in the endoplasmic reticulum (ER) of the host gastrodermal cells. A transcriptomic analysis of genes related to lipogenesis indicated that binding immunoglobulin protein (BiP) plays a key role in metabolic signaling pathways. The diel rhythmicity of LB biogenesis was correlated with ER-localized BiP expression. BiP expression peaked during the period with the largest increase in LB formation, thereby indicating that the chaperoning reaction of abnormal protein folding inside the host ER is likely involved in LB biosynthesis. These findings suggest that the host ER, central to LB formation, potentially facilitates the regulation of endosymbiosis between coral hosts and Symbiodiniaceae. 


\section{The characteristics of host lipid body biogenesis}

G।
$Y e$ 2 during coral-dinoflagellate endosymbiosis

3

4

\section{5}

6

7

\section{Hung-Kai Chen ${ }^{1}$, Sabrina L. Rosset ${ }^{1}$,Li-Hsueh Wang ${ }^{1,2}$, and Chii-Shiarng Chen ${ }^{1,2,3 *}$} (1)

${ }^{1}$ National Museum of Marine Biology and Aquarium, Pingtung, Taiwan

${ }^{2}$ Graduate Institute of Marine Biology, National Dong-Hwa University, Pingtung, Taiwan

${ }^{3}$ Department of Marine Biotechnology and Resources, National Sun Yat-sen University, Kaohsiung, Taiwan

Corresponding Author:

Chii-Shiarng Chen

2 Houwan Road, Checheng, Pingtung 944, Taiwan.

Email address: cchen@nmmba.gov.tw

\section{Abstract}

Intracellular lipid body (LB) biogenesis depends on the symbiosis between coral hosts and their Symbiodinaceae. Therefore, understanding the mechanism(s) behind LB biosynthesis in corals can portentially elucide the drivers of cellular regulation during endosymbiosis. This study assessed LB formation in the gastrodermal tissue layer of the hermatypic coral Euphyllia glabrescens. Diel rhythmicity in LB size and distribution was observed; solar irradiation onset at sunrise initiated an increase in LB formation, which continued throughout the day and peaked after sunset at 18:00. The LBs migrated from the area near the mesoglea to the gastrodermal cell border near the coelenteron. Micro-LB biogenesis occurred in the endoplasmic reticulum (ER) of the host gastrodermal cells. A transcriptomic analysis of genes related to lipogenesis indicated that binding immunoglobulin protein (BiP) plays a key role in metabolic signaling pathways. The diel rhythmicity of LB biogenesis was correlated with ER-localized BiP expression. BiP expression 
31 peaked during the period with the largest increase in LB formation, thereby indicating that the 32 chaperoning reaction of abnormal protein folding inside the host ER is likely involved in LB 33 biosynthesis. These findings suggest that the host ER, central to LB formation, potentially 34 facilitates the regulation of endosymbiosis between coral hosts and Symbiodiniaceae.

35

36

37

38

39

40

41

42

43

44

45

46

47

48

49

50

51

52

53

54

55

56

57

58

59

60

61

\section{Introduction}

Endosymbiosis between coral hosts and dinoflagellates of the Symbiodiniaceae family is of global importance because it serves as the energenic foundation for all coral reefs. However, systematic understanding of how this association functions at the cellular and molecular levels is lacking. A hallmark of symbiosis is the daily rhythmic lipid body (LB) formation and subsequent degradation within the gastrodermal cells of coral hosts (Chen et al., 2012). Metabolic exchange is the basis of coral-algal endosymbiosis, and we previously proposed that these LBs function as centers of this metabolic dialog (Chen et al., 2017). Therefore, LB formation is a compelling target for further research aimed at eucidating mechanisms of endosymbiosis.

LBs are ubiquitous organelles, thought to originate in specialized compartments of the smooth endoplasmic reticulum (ER) (as shown in eukaryotes, see Wilfling et al., 2014) and later bud off into the cytosol. This hypothesis is based on the location of key enzymes involved in storage lipid biosynthesis in the ER and the discovery of ER-specific proteins in LBs (Jacquier et al., 2011). According to the model proposed by Wilfling et al. (2014), storage lipids accumulate between sheaths of the ER membrane, and the recruitment of enzymes (involved in lipid metabolism) and accessory proteins (involved in LB budding and maturation) to the LB formation site results in LB growth. Whether mature LBs completely separate from or remain connected to the ER may vary across biological systems.

Binding immunoglobulin protein (BiP), also known as glucose-regulated protein 78 (GRP78), is an ER-specific protein and a member of the heat shock 70 protein family. Moreover, it is a central regulator of unfolded protein response (UPR) activation to ER stress (Bertolotti et al., 2000) and is associated with purified coral LBs and other lipid droplets (Peng et al., 2011; Prattes et al., 2000; Zhang et al., 2016). Furthermore, by dynamically interacting with organelles such as the Golgi apparatus, mitochondria, endosomes, lysosomes, and lipid droplets through membrane contact sites (Cohen, Valm \& Lippincott-Schwartz, 2018; Wu, Carvalho \& Voeltz, 2018), the ER is generally thought to be involved in not only conveying crucial cellular signals but 
62 also lipid metabolism (Han \& Kaufman, 2012).

63 Light irradiation drives daily LB formation in corals, suggesting that photosynthetically

64 fixed carbon is translocated to coral hosts and incorporated into the following storage lipids, 65 constituting the cores of the LBs: triacylglycerols, sterol esters, and wax esters (Chen et al., 2012). 66 Analyses of the lipidomic profiles of dinoflagellates, LBs, and host gastrodermal cells have 67 suggested that hosts and endosymbionts both contribute fatty acid (FA) moieties to LB 68 biosynthesis (Chen et al., 2017). Furthermore, a study (Chen et al., 2012) revealed that LB density 69 peaks at sunset and returns to baseline overnight; the study also demonstrated that LBs migrate 70 across the host gastroderm during the diel cycle, and the highest densities were observed near the 71 mesoglea at noon and near the coelenteron at night. However, the in situ tissue architecture of coral tentacles used in the study was highly distorted because amputated tentacles were contracted.

Here, we tested the hypothesis that LB biosynthesis in corals occurs in the host ER. We reconfirmed this LB migration trajectory by using an improved protocol, and characterized the process of coral LB maturation using transmission electron microscopy (TEM) to analyze diel fluctuations in LB size and distribution. In addition, transcriptomic data related to UPR activation were used to elucidate the correlation among BiP, ER, and lipogenesis throughout the diel cycle. Complemetary to the transcriptomic-level analysis, LB biosynthesis in corals was found to occur in the host ER when the abundance of the ER marker protein BiP in isolated LBs was measured. Understanding intracellular LB formation and maturation processes in corals can help to elucidate the mechanisms behind coral-algae endosymbiosis; such information could make tangible contributions to coral lipidomics in general, and more specifically, can be applied to research geared toward understanding and mitigating coral bleaching (i.e., the breakdown of symbiosis).

\section{Materials \& Methods}

\section{Coral husbandry}

Corals (Euphyllia glabrescens) were collected from a reef at the inlet of the Third Nuclear Power Plant in Nanwan Bay, Taiwan (21 $\left.57.376^{\prime} \mathrm{N}, 120^{\circ} 45.291^{\prime} \mathrm{E}\right)$ in 2012. Coral collection was approved by the Kenting National Park Management Office. Ten $\sim$ twelve colonies were cultured over eight years in each 4-ton tanks with flow-through seawater (exchange rate of $\sim 80 \mathrm{~L} / \mathrm{h}$ ) in the husbandry center of the National Museum of Marine Biology and Aquarium (NMMBA). Twentyfour colonies for experiments were maintained under an ambient diel cycle with natural sunlight, 
93 and temperature was maintained at $26.5 \pm 1{ }^{\circ} \mathrm{C}$ using a microprocessor-controlled cooler (First FC94 45, Aquatech, Kaohsiung, Taiwan). Light intensity and water temperature were continuously

97 recorded using a HOBO Pendant ${ }^{\circledR}$ logger placed near the colonies in each tank (UA-002; Onset, Pocasset, MA, USA).

\section{Analysis of in situ LB distribution}

Tentacle samples were collected at the following nine times during one diel cycle: 06:00 (sunrise), 9:00, 12:00 (noon), 15:00, 18:30 (sunset), 21:00, 00:00 (midnight), 03:00, and 05:00. Tentacles were collected from three distinct colonies $(n=3$ tentacles / colony) at each time point. A single polyp was targeted only once to avoid sampling-induced stress. For accurate analysis of LBs, preserving the natural tissue architecture of the expanded tentacle was critical. Sampling the tentacle without using a capillary clip resulted in a sectioned gastroderm exhibiting multiple layers of cells containing endosymbionts (Fig. 1A). We circumvented this limitation by successfully preserving the tissue architecture of the naturally expanded tentacle with a clamping technique marked using solid arrows in Fig. 1B and 1C. To verify the effectiveness of this technique, architectural differences in the gastrodermal tissue of extended and contracted tentacles were assessed by staining LBs with osmium tetroxide, cryosectioning fixed specimens, and imaging sections by using differential interference contrast microscopy (Fig. 1A \& D) as described by Chen et al. (2012). This technique allowed the gastroderm to consist of a single cell layer (Fig. 1D).

\section{Ultrastructure analysis through Transmission Electron Microscope}

Clamped tentacles were amputated using micro scissors and immediately fixed with $2.5 \%$ glutaraldehyde and 2\% paraformaldehyde in $100 \mathrm{mM}$ sodium phosphate containing 5\% sucrose $\left(\mathrm{pH} 7.3\right.$ ) for $2.5 \mathrm{~h}$ at $4^{\circ} \mathrm{C}$ then washed with $100 \mathrm{mM}$ sodium phosphate at $4^{\circ} \mathrm{C}$. Specimens were post fixed in $1 \%$ osmium tetroxide in $50 \mathrm{mM}$ sodium phosphate $(\mathrm{pH} 7.3)$ for $1 \mathrm{~h}$ at $4^{\circ} \mathrm{C}$. Samples and data were then proceeded and analyzed according to previously methods by Chen et al. (2012). Individual LB sizes were determined by measuring their maximum diameter $(\mu \mathrm{m})$, and LBs were categorized into three size classes (micro-LBs: $<1 \mu \mathrm{m}$; mid-LBs: $1-3 \mu \mathrm{m}$; large-LBs: $>3 \mu \mathrm{m}$ ) and two distribution categories (near the mesoglea or near the coelenteron) by using Metamorph 6.3 (Molecular Devices). 
124 Transcriptomic analysis of ER genes related to LB lipogenesis

125 Tentacle collection and RNA extraction

126 Tentacles (stretched to a length of $\sim 3 \mathrm{~cm}$ ) were amputated from polyps of E. glabrescens 127 colonies at approximately sunrise (06:00), noon (12:00), sunset (18:00), and midnight (00:00)

128 using curved surgical scissors. The tentacles were then washed twice with filtered seawater (FSW), 129 frozen in liquid nitrogen, and stored at $-80^{\circ} \mathrm{C}$. De novo transcriptome sequencing (RNA-Seq) 130 using Illumina technology was performed by Welgene Biotech (Taipei, Taiwan). Purified RNA 131 was quantified at $260 \mathrm{~nm}\left(\mathrm{OD}_{600}\right)$ by using an ND-1000 spectrophotometer (Nanodrop 132 Technology, Wilmington, DE, USA) and analyzed using a Bioanalyzer 2100 (Agilent 133 Technologies, USA) with an RNA 6000 LabChip kit (Agilent Technologies, USA). All procedures 134 were performed in accordance with manufacturer protocols.

135

136

Sequencing and transcriptome assembly

137 Libraries of all samples were created using the SureSelect Strand-Specific RNA Library Prep 138 kit for $150 \mathrm{PE}$ bp sequencing on the Solexa platform. Sequencing was performed using the TruSeq 139 SBS Kit. Raw sequences were obtained using the Illumina Pipeline software bcl2fastq v2.0, and 140 we expected to generate $2 \mathrm{~Gb}$ per sample. Trimmomatics was then used to generate qualified reads 141 by trimming or removing low-quality bases or reads (QV $\geq 20)$ (Bolger, Lohse \& Usadel, 2014). 142 All qualified $150 \mathrm{PE}$ reads were assembled using the de novo assembly program Trinity (Grabherr 143 et al., 2011). After Trinity assembling, CD-HIT-EST was used to remove contig sequence 144 redundancy ( $\mathrm{Fu}$ et al., 2012). Unigene names were assigned by the partitioning algorithm in the 145 khmer software package (Crusoe et al., 2015). Estimations of unigene abundance were calculated 146 using Cufflinks.

Functional annotation of assembled unigenes

Unigenes in the transcriptome assembly were searched against the National Center for Biotechnology Information nonredundant protein (nr), Gene Ontology (GO), and Kyoto Encyclopedia of Genes and Genomes (KEGG) Pathway databases by using the high-efficiency alignment algorithm RAPSearch2 with a cutoff E value $\leq-3$ (Zhao et al., 2012). The top alignment hits were used to predict the sequence orientations, GO accessions, and related KEGG Pathways 
155 formation was used to understand the distribution of gene functions in the coral host at the macro 156 level. We used the fold change to assess the distributions and variations of genes related to LB 157 lipogenesis for four sampling times.

158

159

160

161

162

163

164

165

166

167

168

169

170

171

172

173

174

175

176

177

178

179

180

181

182

183

184

185

\section{Expression analysis of ER genes related to lipogenesis}

Qualified 150 PE reads were mapped using the short-read alignment software Bowtie2 (Langmead \& Salzberg, 2012). After mapping, the expression estimation of each gene was quantified using Cufflinks (Trapnell et al., 2012). Gene expression levels were calculated as fragments per kilobase of transcript per million mapped reads (FPKM). Furthermore, gene expression levels at different diel-cycle times were calculated as relative expression values of each sampling time. We adopted $\log 2$ (FPKM ratio) as the fold change of gene expression, that is, sunrise $=\log 2($ FPKM sunrise $/$ midnight $)$, noon $=\log 2($ FPKM noon $/$ sunrise $)$, sunset $=\log 2($ FPKM sunset/noon $)$ and midnight $=\log 2$ (FPKM midnight/sunset). FPKM and bioinformatic calculations were also performed by Welgene Biotech (Taipei, Taiwan). JMP v.10 (SAS Institute Inc., Cary, NC, USA) was used to translate the fold changes ( $\log 2$ FPKM) of gene expression and the density distribution of gene expression profiles.

\section{Diel expression pattern of BiP in host cells and purified LBs}

\section{Tentacle collection and LB isolation}

Tentacles were sampled at six time points during the diel cycle: 06:00, 12:00, 15:00, 18:00, 21:00, and 00:00. For a given sampling time, a total of 80 tentacles were collected (pooled from multiple colonies). This sampling strategy was repeated for three distinct diel cycles ( $n=3$ different sampling days). After rinsing tentacles with FSW, tentacle tips were removed to prevent interference from nematocysts. The gastroderm was then separated from the epiderm through incubation with $3 \% \mathrm{~N}$-acetylcysteine ( $\mathrm{pH} 8.2$, prepared in artificial seawater) for $1 \mathrm{~h}$ at room temperature (RT) (Peng et al., 2008). LBs samples and data were then proceeded and analyzed as previously described (Chen et al., 2012; Chen et al., 2017; Peng et al., 2011).

\section{Fluorescence staining of purified $L B$}

Lipid-specific fluorescent dye BODIPY 493/503 (Thermo Fisher Scientific) was prepared in ethanol as a $3.82 \mathrm{mM}(1 \mathrm{mg} / \mathrm{mL})$ stock. Each specimen was stained for 20 min with a working 
186 solution that was a 1:100 dilution of the stock (Gocze \& Freeman, 1994). A fluorescein 187 isothiocyanate (FITC) filter was used for green fluorescence imaging. The ER-specific staining 188 dye ER-Tracker Blue-White DPX (Thermo Fisher Scientific) was prepared in a $1 \mathrm{mM}$ dimethyl 189 sulfoxide stock. LB specimens were stained for $1 \mathrm{~h}$ using a working solution that was a 1:250 190 dilution of the stock (Diwu et al., 1997). A 4',6-diamidino-2-phenylindole filter and FITC filter 191 were used for blue and green fluorescence, respectively.

192

193

194

195

196

197

198

199

200

201

202

203

204

205

206

207

208

209

210

211

212

213

214

\section{Statistical analysis}

\section{Western blotting of BiP}

Host gastrodermal cells were lysed, and LBs were delipidated in accordance with the procedures described by Mastro and Hall (1999) by adding a delipidation solution (tributyl phosphate: acetone: methanol, 1:12:1 v/v/v) to the collected fractions at a 14:1 v/v ratio on ice. This was followed by incubation at $-20^{\circ} \mathrm{C}$ overnight. Preprocessing of protein samples for SDSPAGE analysis were proceeded as previously described in Chen et al. (2015)..

Twenty micrograms of each protein sample were subjected to $12 \%$ SDS-PAGE then blotted onto polyvinylidene fluoride membranes (Immobilon-PSQ $0.45 \mathrm{~mm}$; Millipore, Germany). The membranes were incubated in 5\% skim milk in tris-buffered saline, 0.1\% Tween-20 (TBST), 100 $\mathrm{mM}$ Tris (pH 7.6), and $150 \mathrm{mM} \mathrm{NaCl}$ at RT for $1 \mathrm{~h}$. Incubation was then performed with rabbit anti-GRP78/BiP (ET-21) antibody (1:2000 dilution; cat. no G9043 Sigma-Aldrich) and preimmune serum antibodies (1:5000 dilution) in TBST buffer at $4^{\circ} \mathrm{C}$ overnight. The membranes were then washed five times with TBST buffer for $10 \mathrm{~min}$ each, incubated with horseradishperoxidase-conjugated goat anti-rabbit IgG antibodies (Millipore) in TBST buffer (1:5000 dilution), washed with TBST buffer, and visualized using a SuperSignal West Pico chemiluminescent substrate kit (cat. no 34080, Thermo Fisher Scientific) in accordance with manufacturer recommendations. Experiments were repeated in triplicate, and ImageJ was used to quantify band intensity for protein expression levels (Schneider, Rasband \& Eliceiri, 2012). Relative BiP expression in the LB (or host) fraction was calculated as follows: the ratio of BiP in LB (or host) $=$ the intensity quantification value in the LB (or host) fraction/the total intensity quantification value in the LB and host. 
216

217

218

219 220

221

222

223

224

225

226

227

228

229

230

231

232

233

234

235

236

237

238

239

240

241

242

243

244

245

Data were analyzed using SPSS (version 14.0; SPSS Inc., Armonk, NY, USA). One-way analyses of variance and Tukey pairwise comparisons were conducted to determine the effect of sampling time on LB density, size, location, and gene and protein expression, and results were considered statistically significant when $p$ was $<0.05$. Values are presented as means \pm SDs.

\section{Results}

Ultrastructural and morphological characteristics of host LBs in the gastrodermis

Similar to the biogenesis of lipid droplets in other eukaryotic cells, that of LBs in coralSymbiodiniaceae endosymbiosis has several unique morphological characteristics. We find multiple lines of evidence suggesting that lipid droplets are derived from the ER. First, coral host LBs were in close spatial proximity to mitochondria (Figure 2), endoplasmic reticulum (ER, Figure 2A), and Golgi apparatus (Figure 2D and G), which exhibit high electron density upon transmission electron microscopy (TEM) examination. Our in situ morphology of coral host LBs revealed that they originate in the ER (Fig. 2A) and are surrounded by the ER (Fig. 2B). Fig. 2B and $\mathrm{C}$ illustrates that micro-LBs and some mid-LBs accumulated in the coral host ER and then migrated and fused together. LBs formed a single globule with few inclusion bodies (Figure 2C, D, E and F) and had dense Golgi spots (Fig. 2D and 2G). When fusion occurred, numerous granules with high-electron density (Figure 2E, F, H and I) appeared within the mid-LBs as they fused with micro-LBs, (Fig. 2E and 2H), and when mid-LB fused with large-LB (Fig. 2F and 2I). Finally, large-LBs continued to integrate into larger LBs.

\section{Diel pattern of $\mathrm{LB}$ size and distribution}

To assess the spatiotemporal dynamics of coral LBs during the diel cycle, gastrodermal LBs were analyzed using TEM for three sizes (i.e., micro-LBs: $<1 \mu \mathrm{m}$; mid-LBs: $1-3 \mu \mathrm{m}$; large-LBs: $>3 \mu \mathrm{m}$ ) and two distributions near the mesoglea or coelenteron within the gastroderm (Fig. 3A). Total LBs started to increase at sunrise and gradually increased during the day, reaching maximum density at sunset (Fig. 3B).

Micro-LBs comprised the largest portion of LBs throughout the diel cycle (Fig. 3C; Supplementary Table S1). A significantly larger portion of micro-LBs were located near the 
246 mesoglea (approximately 40-80\%) and they increased moderately throughout the diel cycle (Fig. $2473 \mathrm{C}$; Supplementary Table S1; light period: $37.2 \pm 2.9 \%$ to $44.3 \pm 3.8 \%$; dark period: $40.1 \pm 3.4 \%$ 248 to $53.3 \pm 2.8 \%$ ). The percentage of micro-LBs near the coelenteron gradually decreased from 22.3 $249 \pm 1.7 \%$ at sunrise to $10.0 \pm 3.1 \%$ at sunset (Fig. 3C; Supplementary Table S1) and increased 250 overnight to $13.8 \pm 1.6 \%$ at $21: 00$ and $20.3 \pm 4.2 \%$ at 05:00. The number of mid- and large-LBs 251 near the mesoglea remained relatively low $(0.7 \pm 0.3 \%$ and $13.5 \pm 4.4 \%$, respectively; Fig. $3 \mathrm{C}$; 252 Supplementary Table S1) and nonsignificantly different during the diel cycle (mid-: $p=0.45$; 253 large-: $p=0.11$ ). However, LB populations close to the coelenteron exhibited significant diel 254 fluctuations (mid- and large-: $p<0.05$ ). The percentage of these larger LBs increased during the 255 light period and returned to baseline levels overnight (Fig. 3C). The peak in relative abundance for 256 257 mid- and large-LBs near the coelenteron occurred at 18:30 (23.3 $\pm 1.1 \%)$ and 21:00 (19.3 $\pm 1.7 \%)$, respectively.

258

259

260

261

262

263

264

265

266

267

268

269

270

271

272

273

274

275

276

\section{Transcriptomic analysis of UPR activation/ER stress genes related to lipogenesis}

In response to ER stress, a signal transduction pathway known as UPR is activated, and it may affect the metabolic process of de novo lipid biosynthesis. We analyzed the transcriptome of the coral Euphyllia glabrescens and focused on unigenes and pathways related to LB metabolism.

LB lipogenesis occurs primarily in host cells, where metabolic signals regulate the expression of key enzymes in lipogenic pathways (Fig. 4A, modified from previous models; Morris et al., 1997; Zheng et al., 2010; Basseri \& Austin, 2012). Specifically, as a monitor of UPR activation/ER stress, the ER chaperone and signaling regulator GRP78/BiP exhibited a fourfold increase in expression at noon. Nearly simultaneously, the two sensor proteins protein kinase R-like ER kinase (PERK) and inositol-requiring enzyme 1 (IRE1), which induce various pathways and drive lipid biosynthesis, exhibited near a two-fold increase in expression. IRE1 expression started to increase at sunrise and could reach a two-fold change in transcription factor X-box binding protein 1 (XBP1) expression by noon. The eukaryotic translation initiation factor 2 subunit 1 (eIF2 $\alpha$ ) expression started to increase twofold change at sunrise. Transcription factor sterol regulatory element-binding protein 1 (SREBP1) activated by PERK also had increased expression at noon. The activating transcription factor 6 (ATF6) sensor protein was activated after midnight, whereas membrane-bound transcription factor site-1 protease (S1P) exhibited increased expression at sunset. Fig. 4B presents a box plot of the distributions and outliers of gene expression for UPR 
277 activation/ER stress genes related to LB lipogenesis. Genes including BiP, PERK, eIF2 $\alpha$, 278 SREBP1, IRE1, XBP1, ATF6, and S1P were upregulated starting at sunrise and downregulated 279 from sunset to midnight.

280

281

282

283

284

285

286

287

288

289

290

291

292

293

294

295

296

297

298

299

300

301

302

303

304

305

306

307

\section{Diel fluctuations of ER marker BiP locations}

Fig. 5A illustrates the successful fluorescence staining of purified LBs with lipid marker BODIPY 493/503 and ER-tracker Blue-White dye. The LBs contained neutral lipids and were enclosed in an ER membrane. The aforementioned in situ morphological findings also supported this finding (Fig. 2). BiP was selected to be an ER-specific marker protein to assess the functional association of coral gastrodermal LBs with the host ER. BiP expression was detected only in the coral host and purified LBs but not in endosymbionts (Fig. 5B). Furthermore, the 78-kDa BiP doublet was present in the purified LBs, indicating the close association of BiP with LBs. The two bands of BiPs in the host and purified LBs (Fig. 5B) were removed and analyzed using mass spectrometry (Supplementary Fig. S1). The upper band in both LBs and the host was identified as a 78-kDa glucose-regulated protein (GRP78/BiP), and the lower band in the host fraction was identified as a 73-kDa heat shock protein (HSP70).

BiP expression during the diel cycle was assessed through Western blotting (Fig. 5C) and then quantified (Fig. 5D). To understand the relative ratios of BiP involved in LB lipogenesis of the total host fraction, the diel fluctuations in the relative concentration of $\mathrm{BiP}$ associated with purified LBs were assessed and compared with those of the remaining host fraction (Fig. 5D). The relative expression of $\mathrm{BiP}$ associated with LBs increased significantly at 15:00 and decreased at 18:00 (Fig. 5D). In addition, BiP abundance in the host fraction was significantly higher at night and peaked at 21:00. Thus, BiP exhibited distinct temporal fluctuation patterns in the LB and host fractions.

\section{Discussion}

\section{Cytological characteristics of LB formation reveal LB origin}

We successfully preserved the gastroderm consisting of a single layer of cells (Fig. 1). LBs move to self-assemble and fuse with each other (Boström et al., 2005). This is consistent with our observations that smaller LBs fuse to form larger LBs, resulting in irregularly shaped large LBs (Fig. 2B and C). The fusion of several smaller lipid droplets to larger lipid droplets also contributes 
308 to lipid droplet growth (Guo et al., 2008, Cheng et al., 2009). The LBs were in close spatial 309 proximity to mitochondria, ER, and Golgi apparatus. Originating from the ER, lipid droplets can 310 associate with most other cellular organelles through membrane contact sites (Jacquier et al., 2011;

311 Peng et al., 2011; Olzmann \& Carvalho, 2019). LB mobility was also attributed to the dynamic 312 interactions of LBs with other organelles, including mitochondria (Fig. 2A, D, E and F), ER (Fig. 313 2A, B and C), the Golgi apparatus (Fig. 2D and G), peroxisomes, endosomes, and lysosomes 314 (Herms et al., 2015; Gao \& Goodman, 2015; Crossland, Barnes \& Borowitzka, 1980; Hayes \& 315 Goreau, 1977; Vandermeulen, 1974). Therefore, LBs may play a role in the intracellular trafficking 316 of lipids as well as proteins and other molecules between organelles. Moreover, LBs may migrate 317 within host gastrodermal cells toward intracellular sites of lipid utilization and catabolism. 318 Importantly, LBs biogenesis and degradation, as well as their interactions with other organelles, 319 are tightly coupled to cellular metabolism and facilitate the coordination and communication 320 between different organelles and act as vital hubs of cellular metabolism (Olzmann \& Carvalho, 321 2019). In many cells, lipid droplets undergo active motion, typically along microtubules. This 322 motion has been proposed to aid growth and breakdown of droplets, to allow net transfer of 323 nutrients from sites of synthesis to sites of need and to deliver proteins and lipophilic signals (Pol 324 et al., 2004; Welte, 2009). Thus, nascent LB globule biogenesis likely occurs near the mesoglea. 325 As LBs grow and mature, they migrate across gastrodermal cells and fuse to form large LBs 326 adjacent to the coelenteron.

327 The time-dependent LB relocation may occur inside a single gastrodermal cell during their 328 biogenesis which ultimately manifests as a redistribution of LBs across the gastrodermal tissue 329 layer. LBs move along microtubules and relocate within the cytoplasm (Welte, 2009). A proteomic 330 investigation indicated that cytoskeletal proteins and proteins involved in intracellular trafficking 331 were associated with coral LBs (Peng et al., 2011). The mechanism by which LB populations 332 migrate across the gastroderm to cause this redistribution remains unknown. By correlating LB 333 size and location throughout the diel cycle in naturally expanded tentacle specimens, a study 334 validated the proposed redistribution of LBs from the mesoglea to the coelenteron (Chen et al., 335 2012). Our data further demonstrated that micro- and mid-sized LBs fuse to form large LBs near 336 the coelenteron. In particular, micro-LBs were the most abundant near the mesoglea, whereas mid337 and large-sized LBs increased in abundance near the coelenteron during the light period (Fig. 3). 
338 This finding was consistent with a previous study suggesting that LBs migrate across the 339 gastroderm during the diel cycle (Chen et al., 2012).

340

341

342

343

344

345

346

347

348

349

350

351

352

353

354

355

356

357

358

359

360

361

362

363

364

365

366

367 368

\section{Transcriptional variation of $\mathrm{BiP}$ is correlated with $\mathrm{LB}$ lipogenesis}

Ultrastructural morphology studies have revealed that lipid droplets are closely associated with the ER membrane (Fig. 2), and proteomic studies of lipid droplets isolated from various cell lines have revealed numerous ER proteins (Chen et al., 2012; Peng et al., 2011; Liu et al., 2004; Brasaemle et al., 2004; Wan et al., 2007). The ER is the main site of the synthesis of lipids that constitute most of the lipid components of all biological membranes, and the ER is highly sensitive to changes in intracellular homeostasis and extracellular stimuli. During the perturbation of ER homeostasis, referred to as ER stress, UPR is activated when misfolded proteins accumulate, when reactive oxygen species (ROS) are produced, or when the accumulation of FAs resulting from lipolysis alter ER membrane lipids (Volmer \& Ron, 2015; Walter \& Ron, 2011; Chitraju et al., 2017). We demonstrated that the increase in BiP gene expression from sunrise to noon (Fig. 4) may reflect a localized increase in BiP in daytime within the ER microdomain involved in LB formation (Fig. 5). BiP is the primary regulator of the UPR and is upregulated in response to ER stress (Bertolotti et al., 2000; Morris et al., 1997). Furthermore, PERK and IRE1 cascade facilitates LB formation and possibly lipogenesis, and the ATF6 branch enhances phospholipid biosynthesis (Zheng et al., 2010; Basseri \& Austin, 2012; Zha \& Zhou, 2012). Our transcriptiomic variation results (Fig. 4B) were also consistent with those of previous studies, which indicated a general increase in total lipids, FA concentration, and density in LBs during the light period followed by a decrease at night (Chen et al., 2012; Chen et al., 2017).

In addition to its core function in lipid formation, the diel cycle may provide a mechanism for managing daily oxidative stress induced by photosynthesizing Symbiodinaceae in coral host tissue (Levy et al., 2006). In addition to ROS production in the ER resulting from oxidative protein folding, this could overwhelm ER homeostasis and induce daily ER stress response (Malhotra \& Kaufman, 2007; Levy et al., 2011; Oakley et al., 2017). LB formation may play a role in alleviating ER stress by sequestering unfolded proteins and transiently storing them for degradation (Ploegh, 2007). This has been supported by the observations of increased LB biogenesis induced by ER stress (Fei et al., 2009; Lee et al., 2012), but this functional link remains uncertain (To et al., 2017). 
369

370

371

372

373

374

375

376

377

378

379

380

381

382

383

384

385

386

387

388

389

390

391

392

393

394

395

396

397

398

399

\section{Translational fluctuations of $\mathrm{BiP}$ revealed $\mathrm{LB}$ origins}

After analyzing diel transcriptional variations and the transcriptomic pathway, we selected the ER marker BiP associated with LBs to elucidate the connection among LB formation, the ER, and the diel cycle. We observed an increase in the relative concentration of BiP associated with LBs in midafternoon (Fig. 5). However, BiP expression in the host fraction continued to increase at night (21:00). The increase in the apparent relative abundance of BiP may be due to an increase in either ER network volume or localized BiP concentration in response to upregulated $\mathrm{BiP}$ expression. Our current data cannot be used to distinguish one cause from the other. Nevertheless, the consistent association of $\mathrm{BiP}$ with $\mathrm{LBs}$ throughout the diel cycle and the diel patterns in $\mathrm{BiP}$ concentrations between the LB and host fractions demonstrated that LBs are closely associated with a specialized domain of the host ER. The peak in LB-associated BiP was associated with an increase in the total volume of LBs (Fig. 5), thereby supporting the involvement of ER expansion in LB biosynthesis. Moreover, the nocturnal increase in host ER BiP but not in LB formation may indicate ER expansion at night. The ER is a dynamic organelle, and its tubular network can expand to meet the demands of ER-based processes, including protein folding, modification, and secretion; lipid metabolism; and calcium homeostasis (Federovitch, Ron \& Hampton, 2005).

A persisting question in LB research is whether LBs ever truly detach from the ER (Mishra et al., 2016). This is particularly relevant considering the substantial migration and fusion of LBs in the coral gastroderm. After the peak in LB-associated BiP occurred in the midafternoon, the relative $\mathrm{BiP}$ concentration decreased at sunset (Fig. 5D). However, total LB volume reaches its highest concentration at sunset (Chen et al., 2012; Chen et al., 2017; Peng et al., 2011). Therefore, larger LBs that dominate at sunset likely detach from the ER or are at least less closely associated with the ER. The continuity of the ER membrane with the LB surface may be maintained permanently with stalk-like projections of the ER membrane that allow for the continued lipid and protein trafficking between the organelles (Jacquier et al., 2011; Zehmer et al., 2009). Moreover, the dynamic fluctuation of BiP expression between the coral host and LBs rules out the possibility that $\mathrm{BiP}$ within the LBs was merely an impurity collected in the isolation process. Rather, our findings suggested that coral gastrodermal LBs are functionally connected to a specialized domain of the host ER.

\section{Dynamic schematic model of LB formation}


400

401

402

403

404

405

406

407

408

409

410

411

412

413

414

415

416

417

418

419

420

421

422

423

424

425

426

427

428

429

430

Coral gastrodermal LBs are highly dynamic organelles, and LB formation is highly associated with coral host ER. Imaging analyses of ultrastructural morphological characteristics and the Western blotting of BiP-ER marker protein distribution supported the model of LB formation (Fig. 6). We developed this model of ER involvement in LB biogenesis to illustrate the distribution of BiP expression between a coral host and LBs (Fig. 6A), resulting in dynamic fluctuations in BiP expression (Fig. 5). Furthermore, we proposed a six-stage process of LB formation and maturation (Fig. 6B). First, micro-LBs bud from the ER surrounded by mitochondria (Fig. 6B [i]). Subsequently, more micro-LBs gather and are surrounded by ER sheaths (Fig. 6B [ii]). In the third stage, micro-LBs fuse into larger LBs with different lipid compositions (Fig. 6B [iii]). This might result in different colors in TEM image analysis. During the diel cycle, LB growth and fusion result in mid-LBs. At the same time, a large population of micro-LBs is maintained (Fig. 6B [iv]); this occurs with dense Golgi spots in particular. Mid-LBs continue to fuse to form large-sized LBs $(>6 \mu \mathrm{m})$. When fusion occurs, numerous "dots" form on vesicles in between the LBs, as illustrated in Fig. 6 [v]. Finally, the large-LBs fuse to form mature LBs, as presented in Fig. 6 [vi].

LB size must be strictly regulated to balance lipid storage and mobilization in response to energetic demand (Yang et al., 2012). In response to excess lipid molecules, a cell can increase either the number of LBs or the size of existing LBs. Changes to LB size alter the LB volume-tosurface-area ratio, which affects the rate of LB catabolism. The formation of large LBs as observed in the coral gastroderm (Fig. 3C) is the most efficient form of lipid storage (Yang et al., 2012). Moreover, micro-LBs potentially function to meet immediate energetic demands because of the rapid availability of lipids for catabolism and oxidation (Ariotti et al., 2012). Therefore, heterogeneity in gastrodermal LBs may reflect diversity in LB functions (Zhang et al., 2016). The overnight disappearance of large LBs was hypothesized to occur because of nocturnal lipid catabolism and utilization (Chen et al., 2012), however, the underlying mechanism is not clear.

\section{Conclusions}

Unique diel rhythmicity in LB biosynthesis, growth, and maturation was observed in coral gastrodermal cells, and these processes may play a key role in regulating coral-Symbiodinaceae endosymbiosis (Chen et al., 2012; Chen et al., 2017; Peng et al., 2011). The daily cycle of LB formation, maturation, and degradation is a cellular process underpinning stable coral- 
431 Symbiodinaceae endosymbiosis (Chen et al., 2012; Peng et al., 2011). By demonstrating that LB 432 biogenesis is localized in the host ER, this study provided new insight into the mechanism of 433 metabolite integration within this symbiosis. LBs are composed of FA moieties with both host and 434 symbiont origins (Chen et al., 2017). Therefore, lipid molecules translocated from the symbiont to 435 the host may be transported to the host ER where they enter storage lipid biosynthetic pathways in 436 the host and become integrated into LBs.

437 In conclusion, the ER plays a central role in endosymbiosis regulation. Our results 438 demonstrated that LBs originate in specialized domains of the host ER (likely localized near the 439 mesoglea) but disassociate from the ER when migrating and fusing to form large LBs near the 440 coelenteron. Consequently, we predicted that the disruption of host ER homeostasis induced by 441 environmental stress can damage endosymbiotic function (Oakley et al., 2017). Further research 442 is required to elucidate the precise mechanisms of LB formation, maturation, and catabolism in 443 corals and to examine the roles, apart from energy homeostasis, that LBs might have in coral444 Symbiodinaceae endosymbiosis.

\section{Acknowledgements}

447 We thank Yi-Jyun Chen for performing the experiments. We thank Dr. Anderson B. Mayfield and 448 Crystal J. McRae for reviewing the manuscript draft. We thank the anonymous reviewers whose 449 comments/suggestions helped improve and clarify this manuscript. This work was supported by a 450 grant from the Ministry of Science and Technology (MOST) of Taiwan (formerly the National 451 Science Council [NSC]; NSC 101-2311-B-291-002-MY3 and MOST 108-2311-B-291-001) to 452 CSC and by intramural funding from NMMBA (99200311).

\section{References}

Ariotti N, Murphy S, Hamilton NA, Wu L, Green K, Schieber NL, Li P, Martin S, and Parton RG. 2012. Postlipolytic insulin-dependent remodeling of micro lipid droplets in adipocytes. Molecular biology of the cell 23(10):1826-1837 DOI 10.1091/mbc.e11-10-0847.

Basseri S, and Austin RC. 2012. Endoplasmic reticulum stress and lipid metabolism: mechanisms and therapeutic potential. Biochemistry research international 2012 DOI $10.1155 / 2012 / 841362$.

Bertolotti A, Zhang Y, Hendershot LM, Harding HP, and Ron D. 2000. Dynamic interaction 
462

463

464

465

466

467

468

469

470

471

472

473

474

475

476

477

478

479

480

481

482

483

484

485

486

487

488

489

490

491

492

of $\mathrm{BiP}$ and ER stress transducers in the unfolded-protein response. Nature cell biology 2(6):326-332 DOI 10.1038/35014014.

Bolger AM, Lohse M, and Usadel B. 2014. Trimmomatic: a flexible trimmer for Illumina sequence data. Bioinformatics 30(15):2114-2120 DOI 10.1093/bioinformatics/btu170.

Boström P, Rutberg M, Ericsson J, Holmdahl P, Andersson L, Frohman MA, Borén J, and Olofsson S-O. 2005. Cytosolic lipid droplets increase in size by microtubule-dependent complex formation. Arteriosclerosis, thrombosis, and vascular biology 25(9):1945-1951 DOI 10.1161/01.ATV.0000179676.41064.d4.

Brasaemle DL, Dolios G, Shapiro L, and Wang R. 2004. Proteomic analysis of proteins associated with lipid droplets of basal and lipolytically stimulated 3T3-L1 adipocytes. Journal of Biological Chemistry 279(45):46835-46842 DOI 10.1074/jbc.M409340200.

Chen HK, Wang LH, Chen WNU, Mayfield AB, Levy O, Lin CS, and Chen CS. 2017. Coral lipid bodies as the relay center interconnecting diel-dependent lipidomic changes in different cellular compartments. Scientific reports 7(1):1-13 DOI 10.1038/s41598-017-02722-Z.

Chen WNU, Kang HJ, Weis V, Mayfield AB, Jiang PL, Fang LS, and Chen CS. 2012. Diel rhythmicity of lipid-body formation in a coral-Symbiodinium endosymbiosis. Coral Reefs 31(2):521-534 DOI 10.1007/s00338-011-0868-6.

Cheng J, Fujita A, and Ohsaki Y. 2009 Quantitative electron microscopy shows uniform incorporation of triglycerides into existing lipid droplets. Histochemistry and Cell Biology 132: 281-291 DOI 10.1007/s00418-009-0615-Z.

Chitraju C, Mejhert N, Haas JT, Diaz-Ramirez LG, Grueter CA, Imbriglio JE, Pinto S, Koliwad SK, Walther TC, and Farese Jr RV. 2017. Triglyceride synthesis by DGAT1 protects adipocytes from lipid-induced ER stress during lipolysis. Cell metabolism 26(2):407-418 DOI e403. 10.1016/j.cmet.2017.07.012.

Cohen S, Valm AM, and Lippincott-Schwartz J. 2018. Interacting organelles. Current opinion in cell biology 53:84-91 DOI 10.1016/j.ceb.2018.06.003.

Crossland C, Barnes D, and Borowitzka M. 1980. Diurnal lipid and mucus production in the staghorn coral Acropora acuminata. Marine Biology 60(2-3):81-90 DOI 10.1007/BF00389151.

Crusoe MR, Alameldin HF, Awad S, Boucher E, Caldwell A, Cartwright R, Charbonneau A, Constantinides B, Edvenson G, Fay S, Fenton J, Fenzl T, Fish J, Garcia-Gutierrez 
493

494

495

496

497

498

499

500

501

502

503

504

505

506

507

508

509

510

511

512

513

514

515

516

517

518

519

520

521

522

523

L, Garland P, Gluck J, González I, Guermond S, Guo J, Gupta A, Herr JR, Howe A, Hyer A, Härpfer A, Irber L, Kidd R, Lin D, Lippi J, Mansour T, McA'Nulty P, McDonald E, Mizzi J, Murray KD, Nahum JR, Nanlohy K, Nederbragt AJ, OrtizZuazaga H, Ory J, Pell J, Pepe-Ranney C, Russ ZN, Schwarz E, Scott C, Seaman J, Sievert S, Simpson J, Skennerton CT, Spencer J, Srinivasan R, Standage D, Stapleton JA, Steinman SR, Stein J, Taylor B, Trimble W, Wiencko HL, Wright M, Wyss B, Zhang Q, Zyme E, and Brown CT. 2015. The khmer software package: enabling efficient nucleotide sequence analysis. F1000Research 4:900-900

DOI 10.12688/f1000research.6924.1.

Diwu Z, Lu Y, Zhang C, Klaubert DH, and Haugland RP. 1997. Fluorescent Molecular Probes II. The Synthesis, Spectral Properties and Use of Fluorescent Solvatochromic Dapoxyl Dyes. Photochemistry and Photobiology 66(4):424-431 DOI 10.1111/j.17511097.1997.tb03168.x.

Federovitch CM, Ron D, and Hampton RY. 2005. The dynamic ER: experimental approaches and current questions. Current opinion in cell biology 17(4):409-414 DOI 10.1016/j.ceb.2005.06.010.

Fei W, Wang H, Fu X, Bielby C, and Yang H. 2009. Conditions of endoplasmic reticulum stress stimulate lipid droplet formation in Saccharomyces cerevisiae. Biochemical Journal 424(1):61-67 DOI 10.1042/BJ20090785.

Fu L, Niu B, Zhu Z, Wu S, and Li W. 2012. CD-HIT: accelerated for clustering the nextgeneration sequencing data. Bioinformatics 28(23):3150-3152 DOI 10.1093/bioinformatics/bts565.

Gao Q, and Goodman JM. 2015. The lipid droplet — a well-connected organelle. Frontiers in cell and developmental biology 3:49 DOI 10.3389/fcell.2015.00049.

Gocze PM, and Freeman DA. 1994. Factors underlying the variability of lipid droplet fluorescence in MA-10 leydig tumor cells. Cytometry 17(2):151-158 DOI 10.1002/cyto.990170207.

Grabherr MG, Haas BJ, Yassour M, Levin JZ, Thompson DA, Amit I, Adiconis X, Fan L, Raychowdhury R, and Zeng Q. 2011. Full-length transcriptome assembly from RNA-Seq data without a reference genome. Nature biotechnology 29(7):644-652 DOI 10.1038/nbt.1883. 
524 Guo Y, Walther TC, Rao M, Stuurman N, Goshima G, Terayama K, Wong JS, Vale RD, 525 Walter P, and Farese RV. 2008. Functional genomic screen reveals genes involved in lipid526 droplet formation and utilization. Nature 453(7195):657-661 DOI 10.1038/nature06928.

527

528

529

530

531

532

533

534

535

536

537

538

539

540

541

542

543

544

545

546

547

548

549

550

551

552

553

554

Han J, and Kaufman RJ. 2016. The role of ER stress in lipid metabolism and lipotoxicity. Journal of lipid research 57(8):1329-1338 DOI 10.1194/j1r.R067595.

Hayes RL, and Goreau NI. 1977. Intracellular crystal-bearing vesicles in the epidermis of scleractinian corals, Astrangia danae (Agassiz) and Porites porites (Pallas). The Biological Bulletin 152(1):26-40 DOI 10.2307/1540724.

Herms A, Bosch M, Reddy BJ, Schieber NL, Fajardo A, Rupérez C, Fernández-Vidal A, Ferguson C, Rentero C, and Tebar F. 2015. AMPK activation promotes lipid droplet dispersion on detyrosinated microtubules to increase mitochondrial fatty acid oxidation. Nature communications 6(1):1-14 DOI 10.1038/ncomms8176.

Jacquier N, Choudhary V, Mari M, Toulmay A, Reggiori F, and Schneiter R. 2011. Lipid droplets are functionally connected to the endoplasmic reticulum in Saccharomyces cerevisiae. Journal of cell science 124(14):2424-2437 DOI 10.1242/jcs.076836.

Langmead B, and Salzberg SL. 2012. Fast gapped-read alignment with Bowtie 2. Nature Methods 9(4):357-359. DOI 10.1038/nmeth.1923.

Lee JS, Mendez R, Heng HH, Yang ZQ, and Zhang K. 2012. Pharmacological ER stress promotes hepatic lipogenesis and lipid droplet formation. American journal of translational research 4(1):102 DOI 10.1194/j1r.R028290.

Levy O, Achituv Y, Yacobi Y, Dubinsky Z, and Stambler N. 2006. Diel 'tuning' of coral metabolism: physiological responses to light cues. Journal of experimental biology 209(2):273-283 DOI 10.1242/jeb.01983.

Levy O, Kaniewska P, Alon S, Eisenberg E, Karako-Lampert S, Bay L, Reef R, RodriguezLanetty M, Miller D, and Hoegh-Guldberg O. 2011. Complex diel cycles of gene expression in coral-algal symbiosis. Science 331(6014):175-175 DOI 10.1126/science.1196419.

Liu P, Ying Y, Zhao Y, Mundy DI, Zhu M, and Anderson RG. 2004. Chinese hamster ovary K2 cell lipid droplets appear to be metabolic organelles involved in membrane traffic. Journal of Biological Chemistry 279(5):3787-3792 DOI 10.1074/jbc.M311945200.

Malhotra JD, and Kaufman RJ. 2007. Endoplasmic reticulum stress and oxidative stress: a 
555

556

557

558

559

560

561

562

563

564

565

566

567

568

569

570

571

572

573

574

575

576

577

578

579

580

581

582

583

584

585

vicious cycle or a double-edged sword? Antioxidants \& redox signaling 9(12):2277-2294 DOI 10.1089/ars.2007.1782.

Mastro R, and Hall M. 1999. Protein delipidation and precipitation by tri-n-butylphosphate, acetone, and methanol treatment for isoelectric focusing and two-dimensional gel electrophoresis. Analytical Biochemistry 273(2):313-315 DOI 10.1006/abio.1999.4224

Mishra S, Khaddaj R, Cottier S, Stradalova V, Jacob C, and Schneiter R. 2016. Mature lipid droplets are accessible to ER luminal proteins. Journal of cell science 129(20):3803-3815 DOI 10.1242/jes.189191.

Morris JA, Dorner AJ, Edwards CA, Hendershot LM, and Kaufman RJ. 1997. Immunoglobulin binding protein (BiP) function is required to protect cells from endoplasmic reticulum stress but is not required for the secretion of selective proteins. Journal of Biological Chemistry 272(7):4327-4334 DOI 10.1074/jbc.272.7.4327.

Oakley CA, Durand E, Wilkinson SP, Peng L, Weis VM, Grossman AR, and Davy SK. 2017. Thermal shock induces host proteostasis disruption and endoplasmic reticulum stress in the model symbiotic cnidarian Aiptasia. Journal of proteome research 16(6):2121-2134 DOI 10.1021/acs.jproteome.6b00797.

Olzmann JA, and Carvalho P. 2019. Dynamics and functions of lipid droplets. Nature reviews Molecular cell biology 20(3): 137-155 DOI 10.1038/s41580-018-0085-Z.

Peng SE, Chen WNU, Chen HK, Lu CY, Mayfield AB, Fang LS, and Chen CS. 2011. Lipid bodies in coral-dinoflagellate endosymbiosis: proteomic and ultrastructural studies. Proteomics 11(17):3540-3555 DOI 10.1002/pmic.201000552.

Peng SE, Luo YJ, Huang HJ, Lee IT, Hou LS, Chen WNU, Fang LS, and Chen CS. 2008. Isolation of tissue layers in hermatypic corals by $N$-acetylcysteine: morphological and proteomic examinations. Coral Reefs 27(1):133-142 DOI 10.1007/s00338-007-0300-4.

Ploegh HL. 2007. A lipid-based model for the creation of an escape hatch from the endoplasmic reticulum. Nature 448(7152):435-438 DOI 10.1038/nature06004.

Pol A, Martin S, Fernandez MA, Ferguson C, Carozzi A, Luetterforst R, Enrich C, and Parton RG. 2004. Dynamic and regulated association of caveolin with lipid bodies: modulation of lipid body motility and function by a dominant negative mutant. Molecular biology of the cell 15(1): 99-110 DOI 10.1091/mbc.e03-06-0368.

Prattes S, Horl G, Hammer A, Blaschitz A, Graier WF, Sattler W, Zechner R, and Steyrer 
586

587

588

589

590

591

592

593

594

595

596

597

598

599

600

601

602

603

604

605

606

607

608

609

610

611

612

613

614

615

616

E. 2000. Intracellular distribution and mobilization of unesterified cholesterol in adipocytes: triglyceride droplets are surrounded by cholesterol-rich ER-like surface layer structures. Journal of cell science 113(17):2977-2989 DOI 10.1016/S0021-9150(00)81077-1.

Schneider CA, Rasband WS, and Eliceiri KW. 2012. NIH Image to ImageJ: 25 years of image analysis. Nature Methods 9(7):671-675 DOI 10.1038/nmeth.2089.

To M, Peterson CW, Roberts MA, Counihan JL, Wu TT, Forster MS, Nomura DK, and Olzmann JA. 2017. Lipid disequilibrium disrupts ER proteostasis by impairing ERAD substrate glycan trimming and dislocation. Molecular biology of the cell 28(2):270-284 DOI 10.1091/mbc.e16-07-0483.

Trapnell C, Roberts A, Goff L, Pertea G, Kim D, Kelley DR, Pimentel H, Salzberg SL, Rinn JL, and Pachter L. 2012. Differential gene and transcript expression analysis of RNA-seq experiments with TopHat and Cufflinks. Nature Protocols 7(3):562-578 DOI 10.1038/nprot.2012.016.

Vandermeulen J. 1974. Studies on reef corals. II. Fine structure of planktonic planula larva of Pocillopora damicornis, with emphasis on the aboral epidermis. Marine Biology 27(3):239249 DOI 10.1007/BF00391949.

Volmer R, and Ron D. 2015. Lipid-dependent regulation of the unfolded protein response. Current opinion in cell biology 33:67-73 DOI 10.1016/j.ceb.2014.12.002.

Walter P, and Ron D. 2011. The unfolded protein response: from stress pathway to homeostatic regulation. Science 334(6059):1081-1086 DOI 10.1126/science.1209038.

Wan HC, Melo RC, Jin Z, Dvorak AM, and Weller PF. 2007. Roles and origins of leukocyte lipid bodies: proteomic and ultrastructural studies. The FASEB Journal 21(1):167-178 DOI 10.1096/fj.06-6711 com.

Welte MA. 2009. Fat on the move: intracellular motion of lipid droplets. Biochemical Society Transactions 37(5):991-996 DOI 10.1042/BST0370991.

Wilfling F, Haas JT, Walther TC, and Farese Jr RV. 2014. Lipid droplet biogenesis. Current opinion in cell biology 29:39-45 DOI 10.1016/j.ceb.2014.03.008.

Wu H, Carvalho P, and Voeltz GK. 2018. Here, there, and everywhere: The importance of ER membrane contact sites. Science 361(6401) DOI 10.1126/science.aan5835.

Yang H, Galea A, Sytnyk V, and Crossley M. 2012. Controlling the size of lipid droplets: lipid and protein factors. Current opinion in cell biology 24(4):509-516 DOI 
617

618

619

620

621

622

623

624

625

626

627

628

629

630

631

\subsection{6/j.ceb.2012.05.012.}

Zehmer JK, Bartz R, Bisel B, Liu P, Seemann J, and Anderson RG. 2009. Targeting sequences of UBXD8 and AAM-B reveal that the ER has a direct role in the emergence and regression of lipid droplets. Journal of cell science 122(20):3694-3702 DOI 10.1242/jcs.054700.

Zha BS, and Zhou H. 2012. ER stress and lipid metabolism in adipocytes. Biochemistry research international 2012 DOI 10.1155/2012/312943.

Zhang S, Wang Y, Cui L, Deng Y, Xu S, Yu J, Cichello S, Serrero G, Ying Y, and Liu P. 2016. Morphologically and functionally distinct lipid droplet subpopulations. Scientific reports 6:29539 DOI 10.1038/srep29539.

Zhao Y, Tang H, and Ye Y. 2011. RAPSearch2: a fast and memory-efficient protein similarity search tool for next-generation sequencing data. Bioinformatics 28(1):125-126 DOI 10.1093/bioinformatics/btr595.

Zheng Z, Zhang C, and Zhang K. 2010. Role of unfolded protein response in lipogenesis. World journal of hepatology 2(6):203 DOI 10.4254/wjh.v2.i6.203. 
Figure 1

Using capillary clips to fix tentacle-tissue architecture

(A) Distribution of symbionts (Sym) and lipid bodies (LBs) in tentacles without capillary-clip fixation (scale $=10 \mu \mathrm{m}$ ). (B) Tentacles processed with (right) and without (left) the capillary clip (right; scale $=1 \mathrm{~cm}$ ). (C) Comparison of tentacles with the capillary clip (solid arrow) with normal tentacle of Euphyllia glabrescens coral (open arrow; scale $=1 \mathrm{~cm}$ ). (D) Distribution of Sym and LBs in tentacles with capillary-clip fixation (scale $=10 \mu \mathrm{m}$ ). 

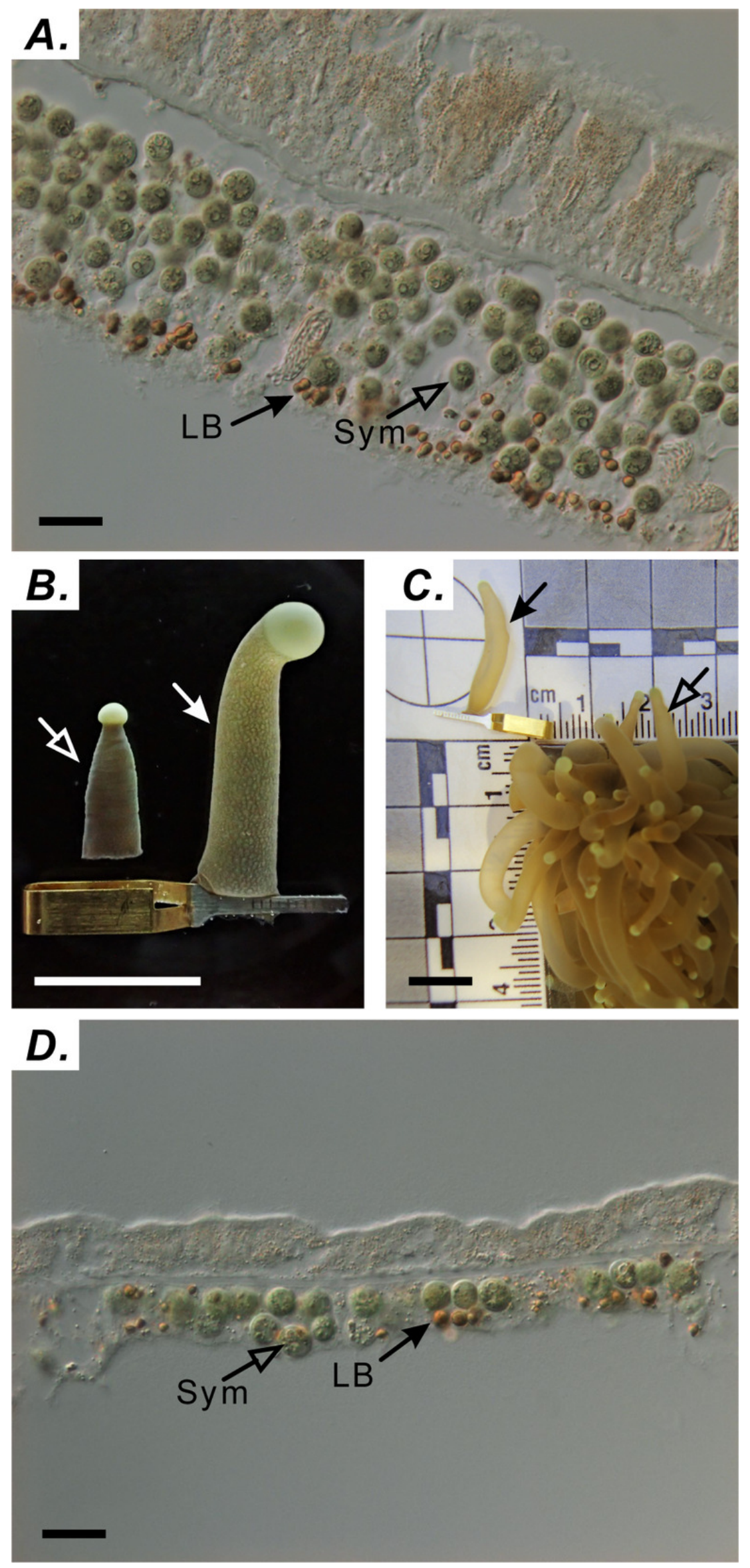


\section{Figure 2}

Diel biosynthesis of host LBs in the gastroderm

(A) LBs from the endoplasmic reticulum (ER, see colored areas, and different colours

represent some fragments of ER) surrounded by mitochondria (red arrows; scale $=0.5 \mu \mathrm{m}$ ).

(B) LBs enveloped by ER (solid red arrows; scale $=0.5 \mu \mathrm{m}$ ). (C) Fused LBs (scale $=0.5 \mu \mathrm{m}$ ).

(D) LBs forming a single globule with several inclusion bodies (scale $=0.5 \mu \mathrm{m}$ ). (E) Singleglobule LB continuing to fuse other micro-LBs with inclusion bodies (see solid yellow arrows, scale $=0.5 \mu \mathrm{m}$ ). High-electron density granules appeared within LBs. (F) Two mid-LBs fused together, and high-electron-density granules (see purple dashed frame) appeared within large-LBs (scale $=1 \mu \mathrm{m}$ ). (G) Enlargement of the red dashed frame in (D) displaying LB fusing near a Golgi apparatus and vesicles (see green dashed frame, scale $=0.2 \mu \mathrm{m})$. $(\mathrm{H})$ Enlargement of the red dashed frame in (E). High-electron-density granules are visible between two fusing LBs (scale $=0.2 \mu \mathrm{m}$ ). (I) Enlargement of the red dashed frame in (F) (scale $=0.2 \mu \mathrm{m}$ ). 

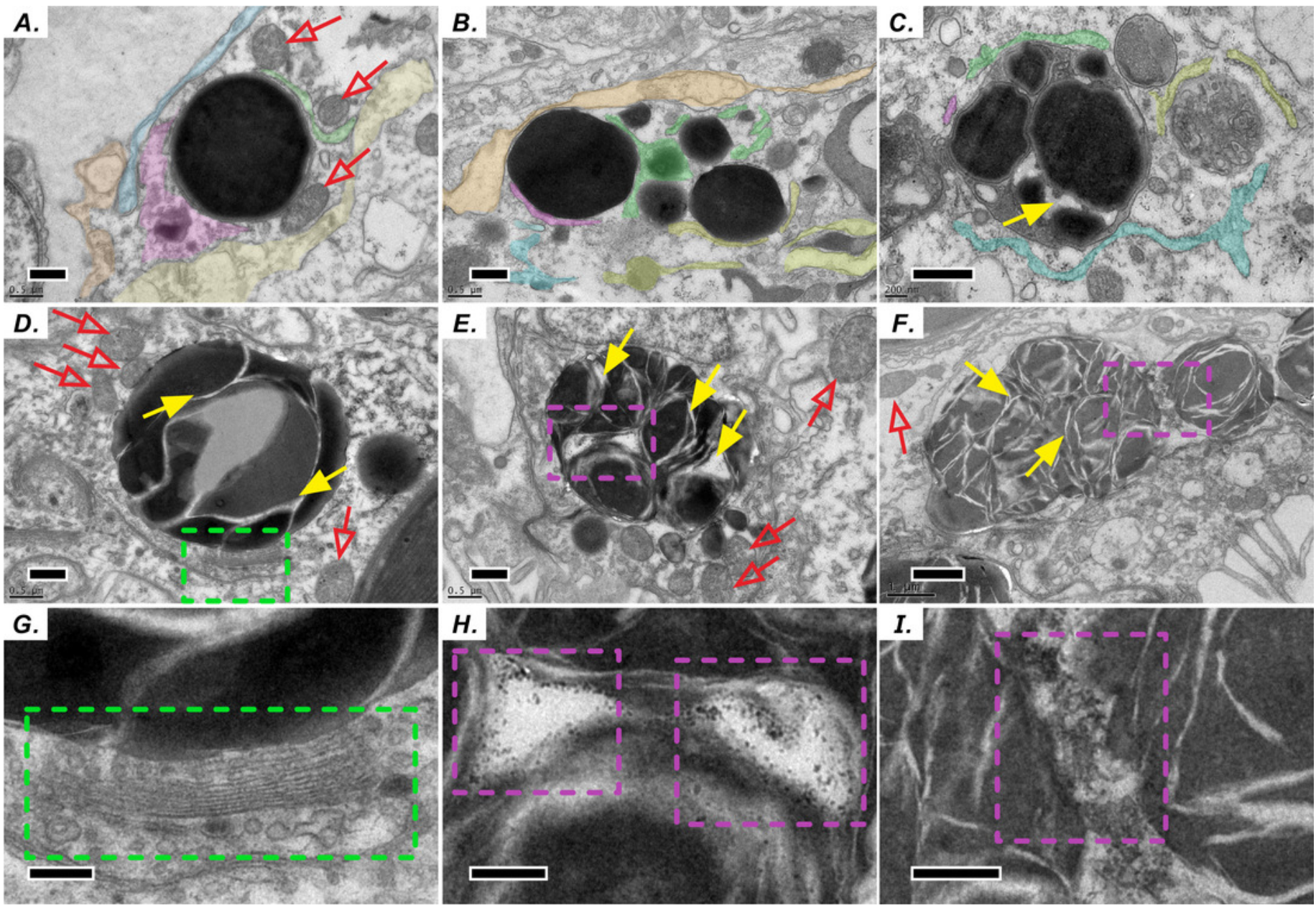


\section{Figure 3}

LB size and distribution during the diel cycle

(A) LB distribution was examined by calculating the number of LBs (red arrow) in two gastrodermal regions: the region near the mesoglea (mes) and the region near the coelenteron (coe; scale $=2 \mu \mathrm{m}$ ). (B) Photosynthetically active radiation and total LBs during a typical diel cycle. (C) Changes in size and distribution of LBs during the diel cycle. Data are presented as mean \pm standard deviation $(\mathrm{n}=3), * p<0.05$, ** $p<0.01$, ${ }^{* * *} p<0.001$ (mesoglea vs. coelenteron; Supplementary Table S1).

A.

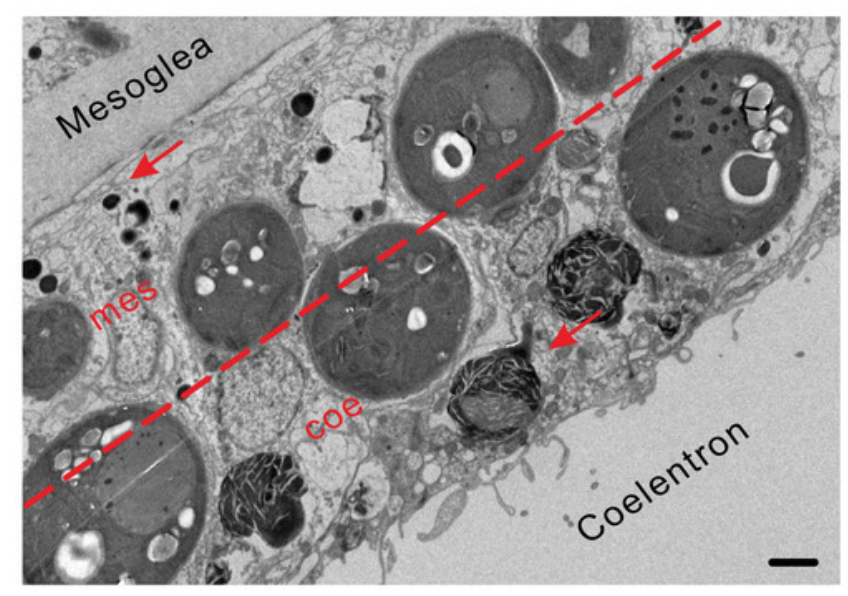

B.

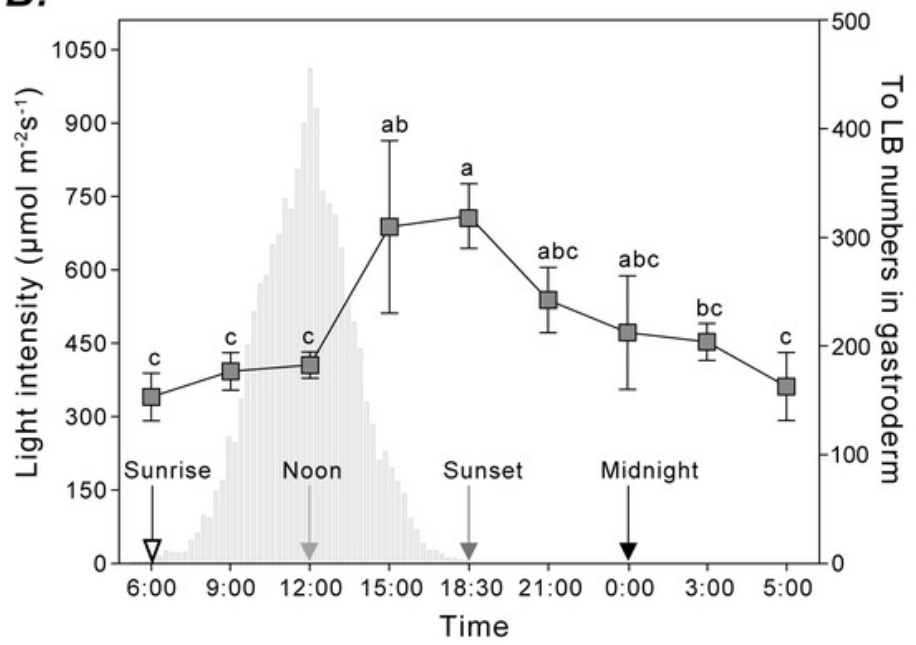

Peer) reviewing PDF | (2021:02:58163:1:1:NEW 25 May 2021)
C.

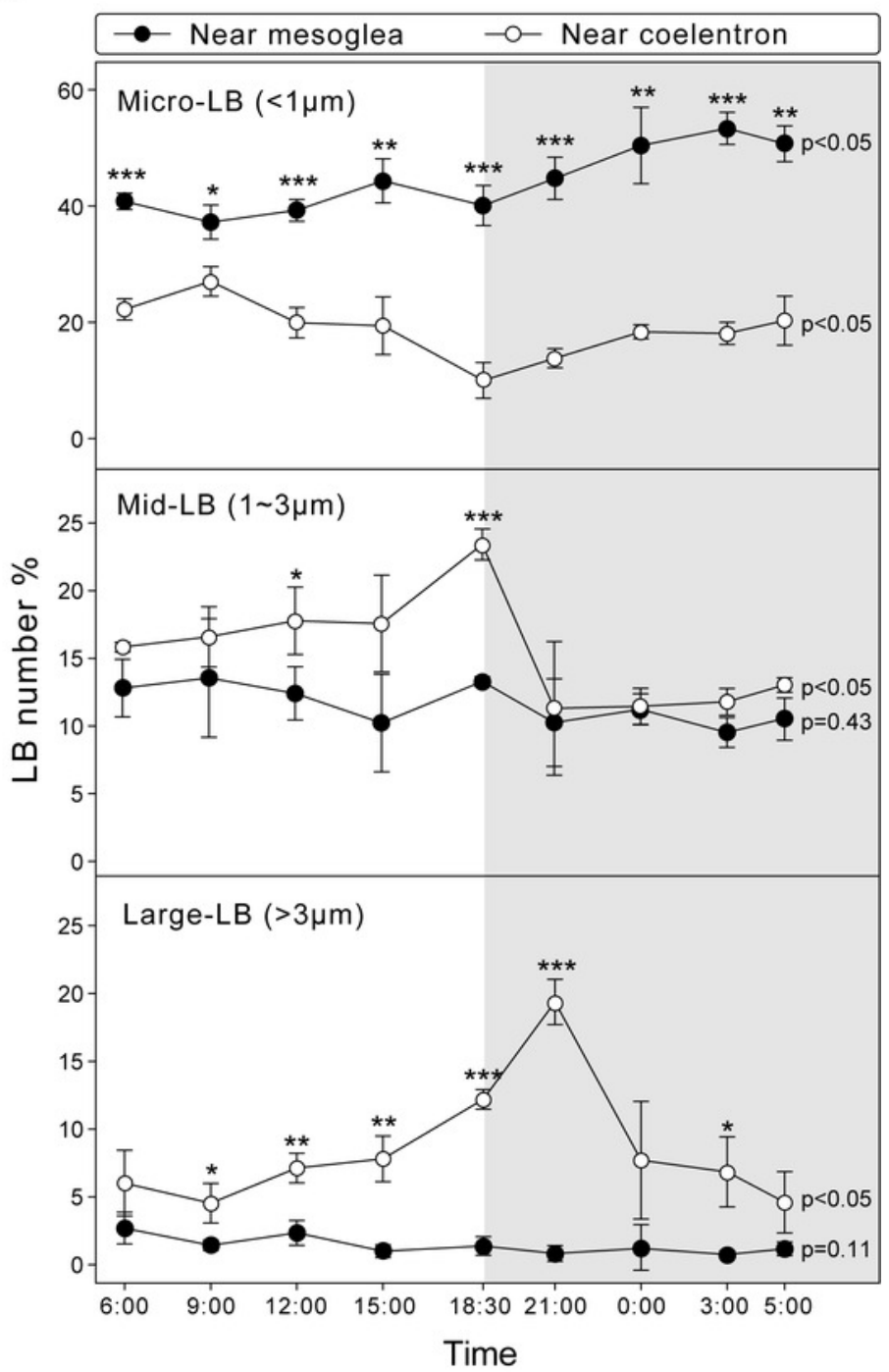




\section{Figure 4}

Transcriptomic analysis of ER stress genes related to LB lipogenesis

(A) ER stress activates the ER stress transducers PERK (left), IRE1 (center), and ATF6 (right) in the ER membrane. The orientation of the colored box corresponds to time points (i.e., sunrise, noon, sunset, and midnight) during the diel cycle. The color scale represents gene fold changes (log2 fragments per kilobase of transcript per million mapped reads) of gene expression (i.e., a red box represents a fourfold increase, and a green box represents a fourfold decrease). (B) Box plot of the distribution and outliers of gene expression levels for UPR activation/ER stress genes related to lipogenesis at sunrise, noon, sunset, and midnight. 
A. Lipogenesis of UPR activation / ER stress

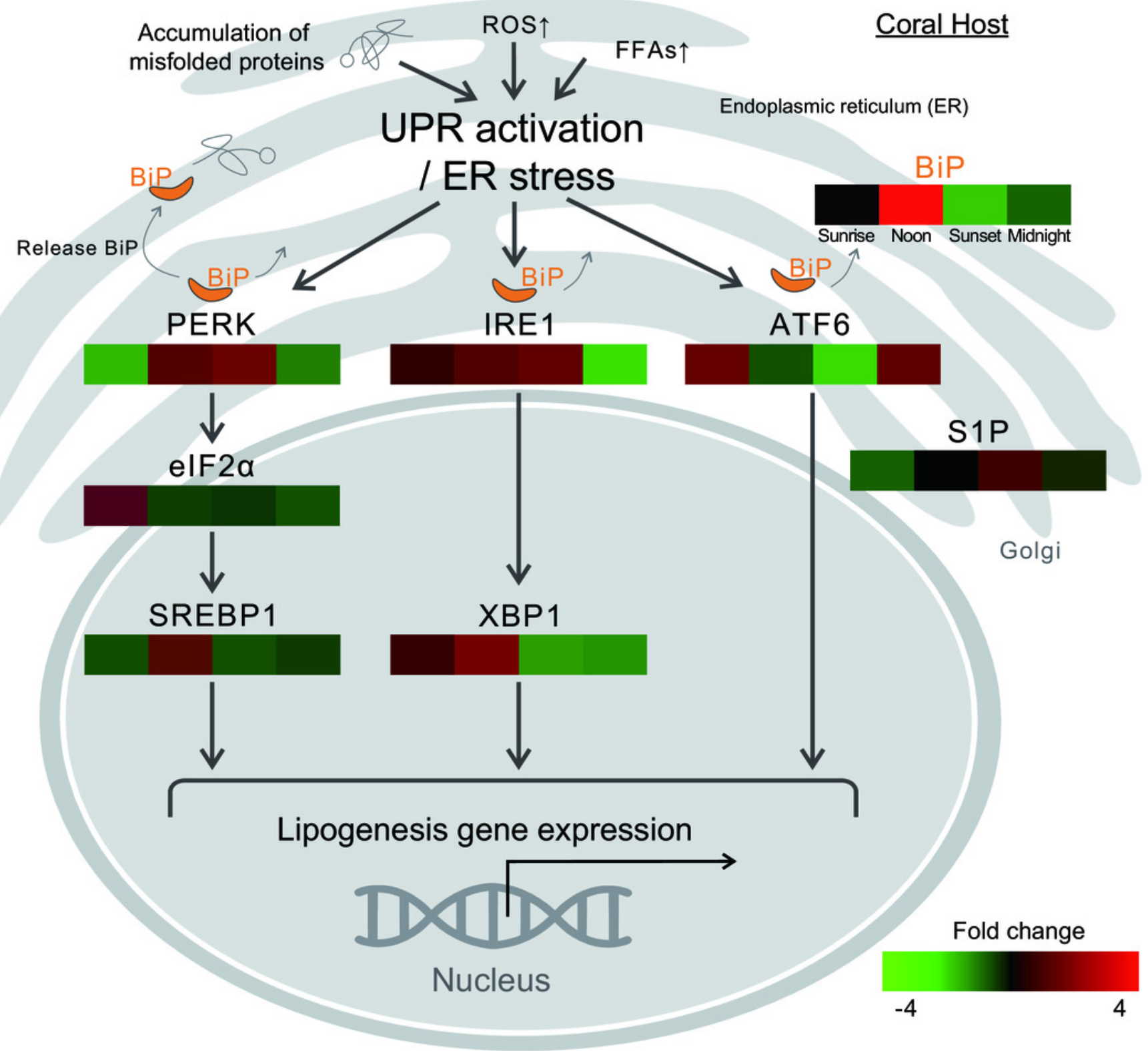

B. Distributions of gene expression values
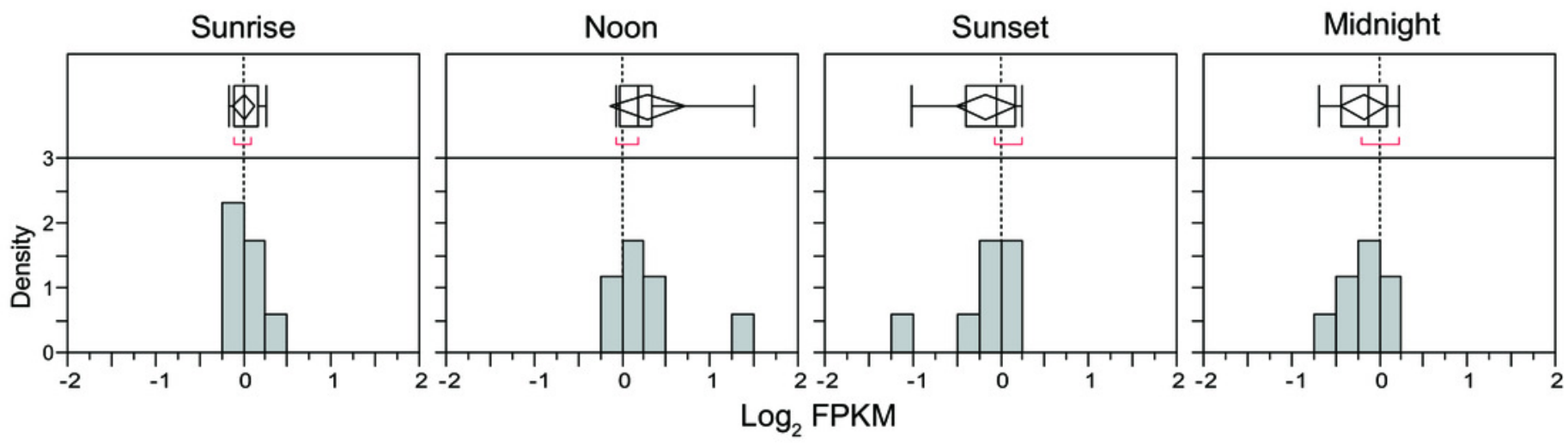


\section{Figure 5}

BiP expression during the diel cycle

(A) [i] Lipid Bodies (LBs) purified from gastrodermal cells under differential interference contrast microscopy. [ii] LBs stained with the neutral lipid marker Bodipy 493/503. [iii] LBs stained with endoplasmic reticulum (ER)-tracker Blue-White dye, which indicated that LBs were enclosed in an ER membrane (scale $=10 \mu \mathrm{m}$ ). See Supplementary Figure S4 for different merging images. (B) BiP expression in the coral host (Host), dinoflagellate endosymbionts (Sym), and isolated LBs after Western blotting with the rabbit anti-BiP antibody. (C) BiP expression in the coral host (Host) and isolated LBs (LBs) during the diel cycle after Western blotting. (D) Further quantification and plotting of blots. See Supplementary Figure S2, S3 and Table S3 for the full-length blots and percentages of ERrelated BiP expression in the host and LB fractions 
A.
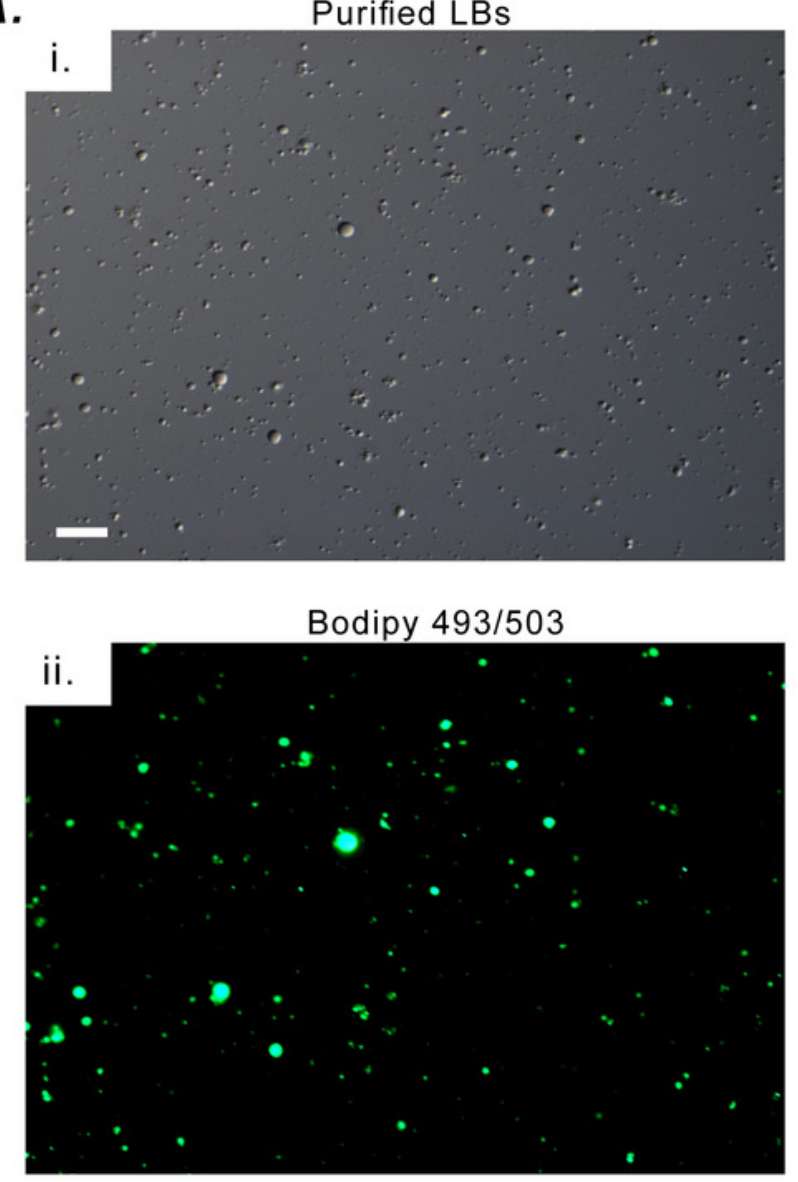

ER-Tracker Blue-White

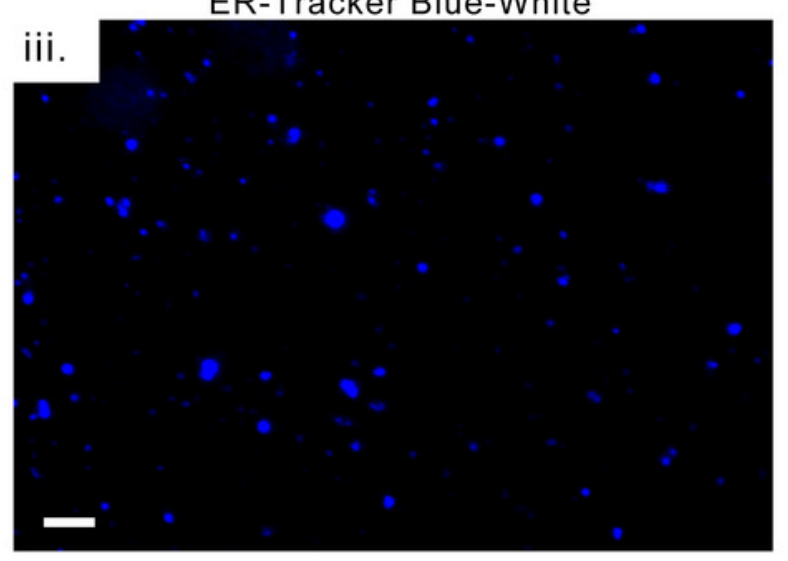

$B$.

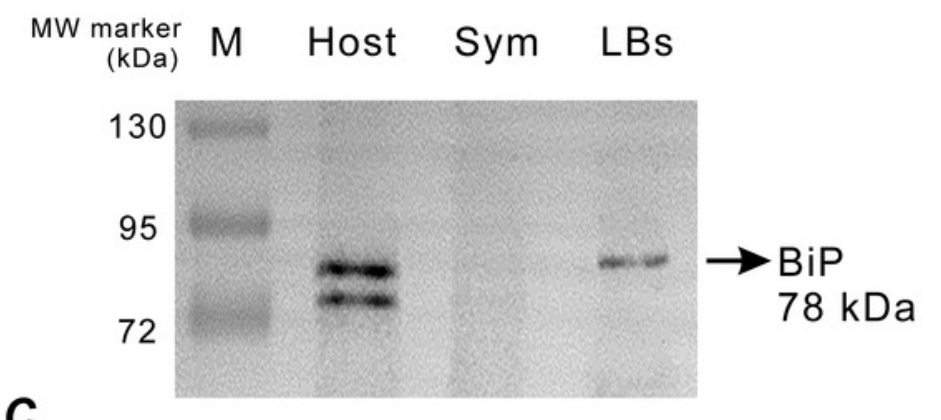

c.

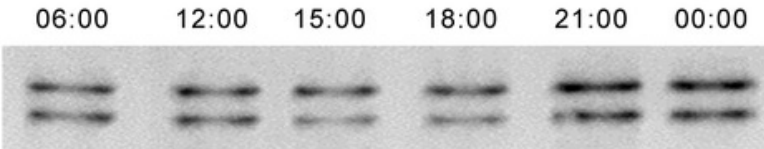

LBs

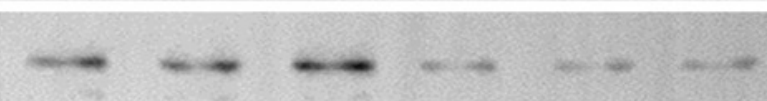

D.

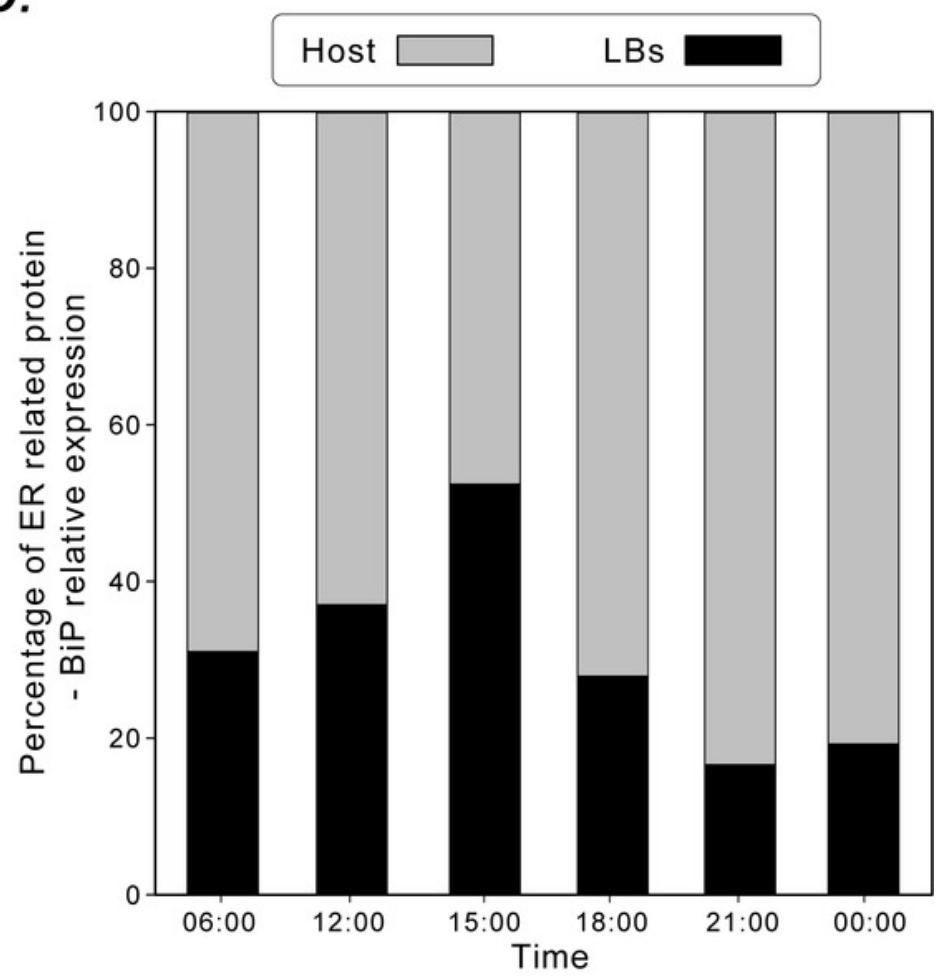




\section{Figure 6}

Schematic summary of LB formation in coral-Symbiodinaceae endosymbiosis

(A) Proposed model of endoplasmic reticulum (ER) involvement in LB biogenesis resulting in the BiP expression distribution between coral host and LBs. (B) LB formation and maturation. [i] LB budding. [ii] ER surrounding. [iii] LB assembling. [iv] Golgi modification. [v] LB fusion. [vi] LB maturation.

A.

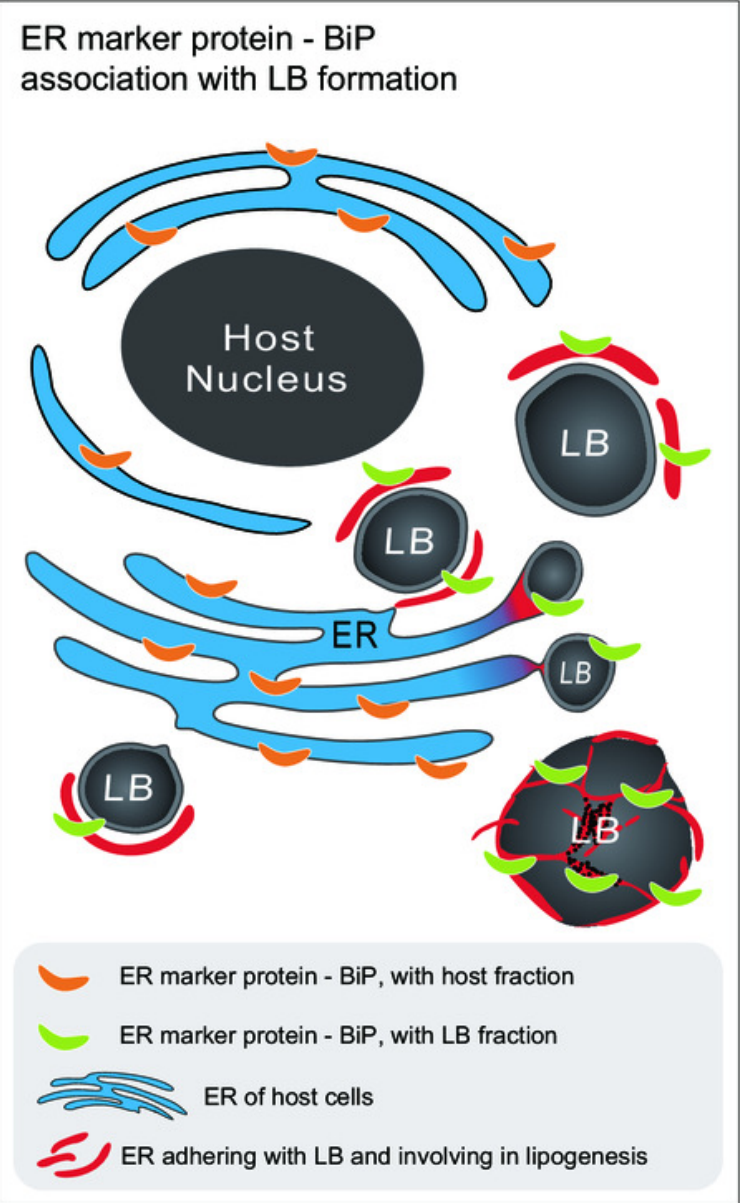

B.

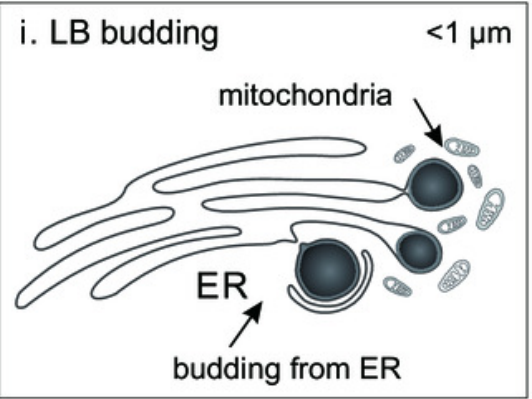

ii. ER surrounding
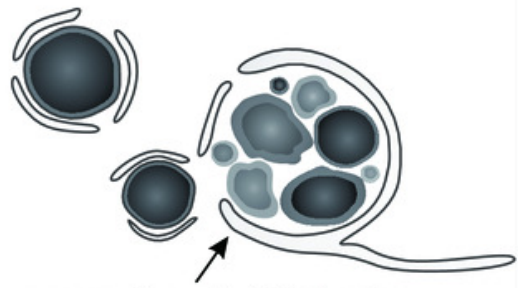

surrounding with ER sheaths

iii. LB assembling

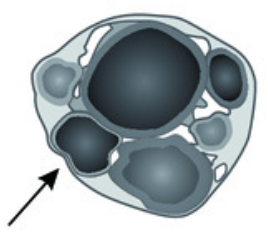

Fusion of small LBs, with different lipid composition

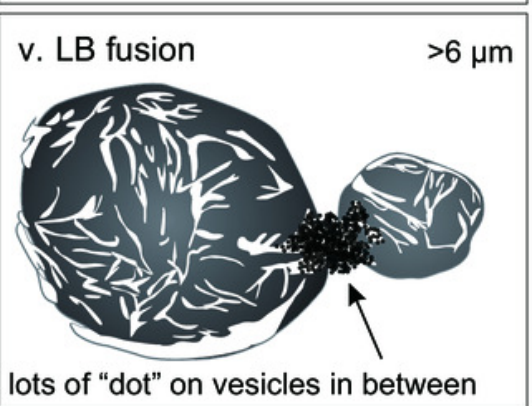

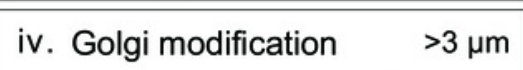

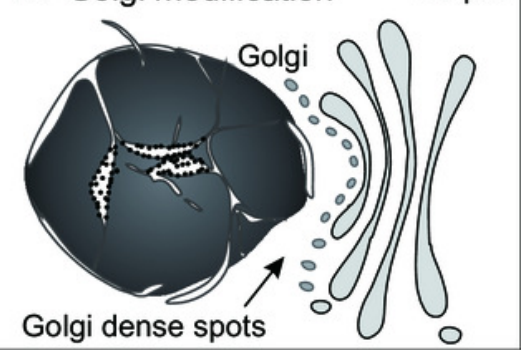

vi. LB maturation

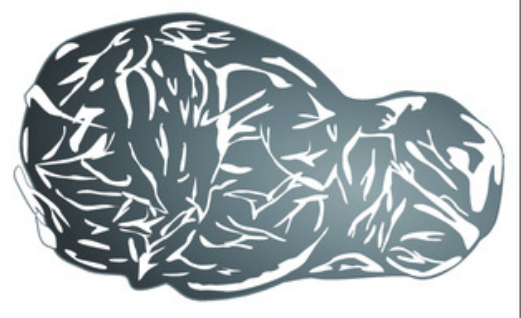

